\title{
Prevalencia de enfermedad renal crónica en centros urbanos de atención primaria
}

\author{
CARLOS ZÚÑIGA SM.,2,3, HANS MÜLLER O.,2,3, MARITZA FLORES O.,a
}

${ }^{1}$ Facultad de Medicina Universidad Católica de la Santísima Concepción, Chile.

${ }^{2}$ Facultad de Medicina Universidad de Concepción, Chile.

${ }^{3}$ Instituto de Nefrología Concepción, Chile. aEstadístico.

Recibido el 7 de marzo de 2011, aceptado el 11 de julio de 2011.

Correspondencia a: Dr. Carlos Zúñiga San Martín Facultad de Medicina. Universidad Católica Ssma. Concepción. Alonso de Ribera 2850 Fono/Fax: 56-41-2331161 E-mail: czunigasm2002@ yahoo.com

\section{Prevalence of chronic kidney disease in subjects consulting in urban primary care clinics}

Background: Chronic kidney disease (CKD) is a major worldwide public health problem and is associated with increased risk of cardiovascular disease and death. Aim: To assess CKD prevalence in urban Primary Care Services (PCS) of Concepcion, Chile. Material and Methods: The clinical records of 27.894 adults aged $55 \pm 18$ years (66\% females), consulting in outpatient clinics and in whom serum creatinine was measured, with or without assessment of urine albumin levels, were reviewed. The glomerular filtration rate (eGFR) was estimated using the Modification of Diet in Renal Disease (MDRD)-4 equation. CKD was defined as an eGFR $<60 \mathrm{ml}$ min/1.73 $\mathrm{m}^{2}$ and classified according to the National Kidney Foundation Kidney Disease Outcomes Quality Initiative (NFK-KDOQI) guidelines. Results: Mean eGFR was $77.1 \pm 16.3 \mathrm{ml} / \mathrm{min} / 1.73 \mathrm{~m}^{2}$. Twelve percent of subjects had CKD (women, $14.5 \%$ and men 7,4\%, $p<0,05)$. The prevalence of stages 3, 4 and 5 of CKD were 11.6, 0.3 and $0.2 \%$ respectively. eGFR was negatively correlated with age $(r=-0,54, p<0,05)$. Among patients with an eGFR $<60 \mathrm{ml} / \mathrm{min} / 1.73 \mathrm{~m}^{2}, 96.3 \%$ had eGFR 30-59, 2.3\% $15-29$ and $1.4 \%<$ of 15 . Seventy nine percent were women. $75.1 \%$ were aged 65 years or more, $26.8 \%$ had a serum creatinine equal or less than $1.0 \mathrm{mg} / \mathrm{dL}$ and $40.5 \%$ had microalbuminuria. Only 1\% of outpatients ascribed to Cardiovascular or Diabetes Programs had the diagnosis of CKD registered. Independent risk predictors of CKD were age $>60$ years, female sex and microalbuminuria. Conclusions: This study showed a high prevalence of CKD in ambulatory patients, mainly among women and older people. The low level of diagnosis of CKD in cardiovascular and diabetes programs is of concern.

(Rev Med Chile 2011; 139: 1176-1184).

Key words: Kidney diseases; Prevalence; Primary health care.
L os indicadores de salud pública a nivel mundial y nacional muestran un alarmante y progresivo aumento del número de pacientes con enfermedad renal crónica (ERC), asociado a la elevada prevalencia de patologías como la hipertensión arterial y la diabetes mellitus ${ }^{1-7}$.

La ERC puede progresar a insuficiencia renal crónica y tiene un mayor riesgo de enfermedad cardiovascular y mortalidad, por tanto, promover la prevención a través del diagnóstico precoz y el tratamiento oportuno de la población en riesgo, permitiría detener o enlentecer la progresión del daño renal y disminuiría su morbimortalidad ${ }^{8-14}$. Para lograr este objetivo e implementar políticas sanitarias que contribuyan a atenuar su impacto en la salud de las personas y disminuir su creciente incidencia, es necesario conocer la prevalencia de la ERC en los distintos grupos de la población.

Se estima que $10 \%$ de la población mundial tiene ERC y $90 \%$ de las personas que la padecen no lo saben ${ }^{14}$. Alrededor de 1,5 millones de pacientes en el mundo están en diálisis o con trasplante renal, los cuales se duplicarán en los próximos 10 años ${ }^{14}$. 
En Chile, los indicadores epidemiológicos señalan una prevalencia de ERC en la población general de 2,7\%, y un incremento del número de pacientes en hemodiálisis crónica de 12,7 pacientes por millón de personas (PMP) en 1980 a 903 PMP en el $2010^{3,4}$. Sin embargo, existe escasa información del número de personas en etapas previas de la enfermedad que se atiende regularmente en la atención primaria de salud.

El objetivo de este estudio fue evaluar la prevalencia de enfermedad renal crónica en población adulta que se atiende en los Centros urbanos de Salud Familiar (CESFAM) de la ciudad de Concepción, perteneciente a la red de atención primaria en Chile.

\section{Pacientes y Método}

Estudio descriptivo de corte transversal multicéntrico de los registros clínicos de pacientes atendidos en los consultorios CESFAM en Concepción, entre enero 2009 y junio 2010. Se incluyeron pacientes $\geq 18$ años, de ambos sexos, que consultaron por cualquier motivo de salud y a quienes su médico tratante solicitó creatinina sérica sola o asociada a albuminuria. Se consignó edad, sexo y si estaba inscrito en el programa cardiovascular de su consultorio, donde se controla a los pacientes con diabetes mellitus (DM) e hipertensión arterial (HTA). Se establecieron 4 grupos por edad: a) < de 40 años; b) 40-59 años; c) 60-69 años y d) $\geq 70$ años.

Se estimó la velocidad de filtración glomerular (VFGe) con la ecuación MDRD-4 del estudio Modification of Diet in Renal Disease ${ }^{15-17}$.

$$
\begin{aligned}
\text { VFG estimado }= & 186 \times\left(\text { creatinina }{ }^{-1,154} \mathrm{x}(\text { edad })^{-0,203}\right. \\
& \mathrm{x}(0,742 \text { si mujer }) . \\
& \left(\text { Expresado en } \mathrm{ml} / \mathrm{min} / 1,73 \mathrm{~m}^{2}\right) .
\end{aligned}
$$

Para evaluar albuminuria se empleó la razón albúmina/creatinina (RAC) en muestra de orina aislada expresada en $\mathrm{mg} / \mathrm{g}$ de creatinina ${ }^{16,18-20}$.

Se utilizó la definición de ERC propuesta por la US NKF-KDOQI (National Kidney FoundationKidney Disease Outcomes Quality Initiative) que considera los siguientes criterios: Una disminución de la función renal, expresada por una velocidad de filtración glomerular (VFG) $<60 \mathrm{~mL} / \mathrm{min} / 1,73 \mathrm{~m}^{2}$ y/o la presencia de daño renal (alteraciones estructurales o funcionales del riñón), independiente de la causa, por 3 meses o más. La combinación de ambos criterios diagnósticos permite clasificar la ERC en 5 etapas de menor a mayor compromiso funcional (Tabla 1). En los estadios iniciales (1 y 2) la VFGe no es diagnóstico por sí misma de ERC y requiere de la presencia de algún marcador asociado de lesión renal. En cambio en los estadios 3 a 5 , es suficiente la VFG $<60 \mathrm{ml} / \mathrm{min} / \mathrm{m}^{2}$ para establecer el diagnóstico de $\mathrm{ERC}^{16,21}$.

\section{Definiciones para este estudio}

a) Enfermedad Renal Crónica (ERC): Una VFGe $<60 \mathrm{ml} / \mathrm{min} / 1,73 \mathrm{~m}^{2}$, lo cual comprende las Etapas 3 (VFGe 30-59), 4 (VFGe 15-29) y 5 (VFGe $<15)$ de la ERC. La Etapa 3 de la ERC se dividió en los subgrupos 3 A (VFGe 45-59) y 3 B (VFGe 30-44).

b) Enfermedad Renal Crónica Oculta: Un VFGe $<60 \mathrm{ml} / \mathrm{min} / 1,73 \mathrm{~m}^{2}$ con una creatinina sérica $\leq 1,0 \mathrm{mg} / \mathrm{dl}$.

c) Microalbuminuria: Una RAC entre 30 y 300 $\mathrm{mg} / \mathrm{g}^{7,12-13}$.

d) Albuminuria clínica o macroalbuminuria cifras superiores a $300 \mathrm{mg} / \mathrm{g}$.

Todos los exámenes se procesaron en el Laboratorio Clínico de la Dirección de Salud Municipal de Concepción. La creatinina se determinó en sangre y orina con el método Jaffé sin desproteinización y la albuminuria con inmuno ensayo turbidimétrico. Ambos con un equipo Hitachi 917 con reactivos Diasys ${ }^{\circledR}$. El coeficiente inter ensayo fue $2,95 \%$ para creatinina y $4,45 \%$ para albuminuria.

Tabla 1. Clasificación de enfermedad renal crónica

\begin{tabular}{|clc|}
\hline Etapa & Descripción & $\begin{array}{c}\text { VFG } \\
\left(\mathbf{m l} / \mathbf{m i n} / \mathbf{1 , 7 3} \mathbf{~ m}^{\mathbf{2}}\right)\end{array}$ \\
\hline 1 & $\begin{array}{l}\text { VFG normal con daño } \\
\text { renal }\end{array}$ & $\geq 90$ \\
2 & $\begin{array}{l}\text { VFG levemente reducida } \\
\text { con daño renal }\end{array}$ & $60-89$ \\
3 & $\begin{array}{l}\text { VFG moderadamente } \\
\text { reducida }\end{array}$ & $30-59$ \\
4 & $\begin{array}{l}\text { VFG severamente reducida } \\
\text { con daño renal }\end{array}$ & $15-29$ \\
5 & Falla renal "terminal" & $<15$ (Diálisis) \\
\hline
\end{tabular}

ERC: Enfermedad renal crónica; VFG: Velocidad de filtración glomerular. 
La población asignada a los consultorios de Salud Municipal de Concepción es 112.100 habitantes, de los cuales 79.591 (71\%) son mayores de 18 años). Un total de 17.290 pacientes están registrados en los programas de salud cardiovascular en los 6 consultorios CESFAM.

\section{Análisis estadístico}

Para el análisis estadístico, se utilizó el software SPSS 16.0. La descripción de variables se hizo a través de la determinación de proporciones, promedios y desviaciones estándar. Los análisis bivariados se efectuaron a través de los test $\chi^{2}$ para independencia de variables, $t$-test para muestras independientes, cálculo del coeficiente de correlación lineal "r" de Pearson. Se utilizó test de Kolmogorov-Smirnov para verificar normalidad en la distribución de las variables cuantitativas. Finalmente, se efectuó análisis de Regresión Logística binaria. Se consideraron significativas diferencias con un $\mathrm{p}<0,05$.

\section{Resultados}

Se estudió 29.184 pacientes adultos en los CESFAM, a quienes por indicación médica se realizó determinaciones de creatinina sérica sola o asociada a albuminuria. Se excluyó del análisis a 1.290 pacientes $(4,4 \%)$ por información insuficiente.

La Tabla 2 presenta las características generales de los 27.894 pacientes estudiados: 66\% mujeres; edad promedio 55,5 \pm 17,6 (rango: 18-102 años). De ellos, 16.318 pacientes $(58,5 \%)$ se controlaban en el programa cardiovascular de su consultorio, lo cual corresponde al 94,4\% del total de personas registrados en estos programas.

En 5.718 pacientes $(21 \%)$ se midió simultáneamente albuminuria. El promedio de creatinina sérica fue $0,9 \pm 0,3 \mathrm{mg} / \mathrm{dL}$; la VFGe $77 \pm 1 \mathrm{ml} /$ $\mathrm{min} / 1,73 \mathrm{~m}^{2}$ y la RAC $38 \pm 94 \mathrm{mg} / \mathrm{gr}$, todos ellos con valores significativamente mayores en hombres ( $\mathrm{p}<0,0001)$.

La prevalencia global de ERC fue del 12,1\% (3.371 pacientes), significativamente superior en mujeres que en hombres, $14,5 \%$ vs $7,4 \%$ respectivamente $(\mathrm{p}<0,05)$. El 11,6\% estaba en Etapa 3; $0,3 \%$ en Etapa 4 y 0,2\% en Etapa 5. No se incluyó pacientes en diálisis.

La prevalencia de ERC aumentó en forma significativa de menor a mayor edad, siendo $0,9 \%$ en $<$ de 40 años; $4,5 \%$ entre $40-59 ; 13,5 \%$ entre $60-69$ y $31,8 \%$ en $\geq 70$ años ( $<<0,0001)$ (Tabla 3 , Figura 1). Hubo una significativa correlación negativa entre edad y la VFGe (Pearson $r=-0,54) \mathrm{p}<0,05$.

Del total de pacientes con VFGe $<60,96,3 \%$ estaban en Etapa 3(81,5\% y $14,8 \%$ en subgrupo

Tabla 2. Características generales de los pacientes

\begin{tabular}{|c|c|c|c|c|}
\hline & Hombres & Mujeres & Total & \\
\hline $\mathrm{N}^{\circ}$ de pacientes ${ }^{\mathrm{a}}$ & $9.539(34)$ & $18.355(66)$ & 27.894 & \\
\hline Pacientes en PCVb & $5.548(34)$ & $10.770(66)$ & 16.318 & \\
\hline Edad` (años) & $56(17)$ & $55(18)$ & $55,5(18)$ & \\
\hline \multicolumn{5}{|l|}{ Grupos de edada } \\
\hline$<40$ años & $1.630(31)$ & $3.691(69)$ & $5.321(19)$ & \\
\hline 40 - 59 años & $3.464(34)$ & $6.819(66)$ & $10.283(37)$ & \\
\hline 60 - 69 años & $2.157(38)$ & $3.565(62)$ & $5.722(21)$ & \\
\hline 70 y más años & $2.288(35)$ & $4.280(65)$ & $6.568(23)$ & \\
\hline Laboratorio & & & & $\mathbf{p}$ \\
\hline Creatinina $(\mathrm{mg} / \mathrm{dL})^{c}$ & $1,1(0,4)$ & $0,9(0,3)$ & $0,9(0,3)$ & 0,0001 \\
\hline FGe (MDRD-4) $)^{c, d}$ & $83(17)$ & $74(15)$ & $77(16)$ & 0,0001 \\
\hline $\operatorname{RAC}^{c, e}(n=5.718)$ & $43(108)$ & $35(84)$ & $38(94)$ & 0,0001 \\
\hline
\end{tabular}

avalores expresados como número total de casos y porcentaje entre paréntesis. ${ }^{b} \mathrm{PCV}$ : Programa cardiovascular. 'Valores expresados como media y desviación estándar entre paréntesis. ${ }^{\mathrm{F}} \mathrm{FGe} .(\mathrm{MDRD}-4)$ : Filtrado glomerular estimado expresado en $\mathrm{ml} /$ min/1,73. eRAC: Razón albúmina/creatinina en orina aislada. (mg/gr) medida en 5.718 pacientes. 
Tabla 3. Prevalencia de ERC* según grupos de edad y género en relación al total de pacientes estudiados

\begin{tabular}{|cccc|}
\hline Grupos de edad (años) & Hombres con ERC & Mujeres con ERC & Total con ERC $^{\mathbf{a}}$ \\
\hline$<40$ & $7(0,4)$ & $41(1,1)$ & $48(0,9)^{\mathrm{b}}$ \\
\hline $40-59$ & $85(2,5)$ & $374(5,5)$ & $459(4,5)^{\mathrm{b}}$ \\
\hline $60-69$ & $161(7,5)$ & $614(17,2)$ & $775(13,5)^{\mathrm{b}}$ \\
\hline 70 y más & $454(19,8)$ & $1.635(38,2)$ & $2.089(31,8)^{\mathrm{b}}$ \\
\hline Total & $707(7,4)$ & $2.664(14,5)$ & $3.371(12,1)$ \\
\hline
\end{tabular}

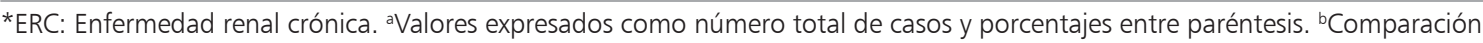
$\%$ entre los grupos de edad y género $p<0,05$.

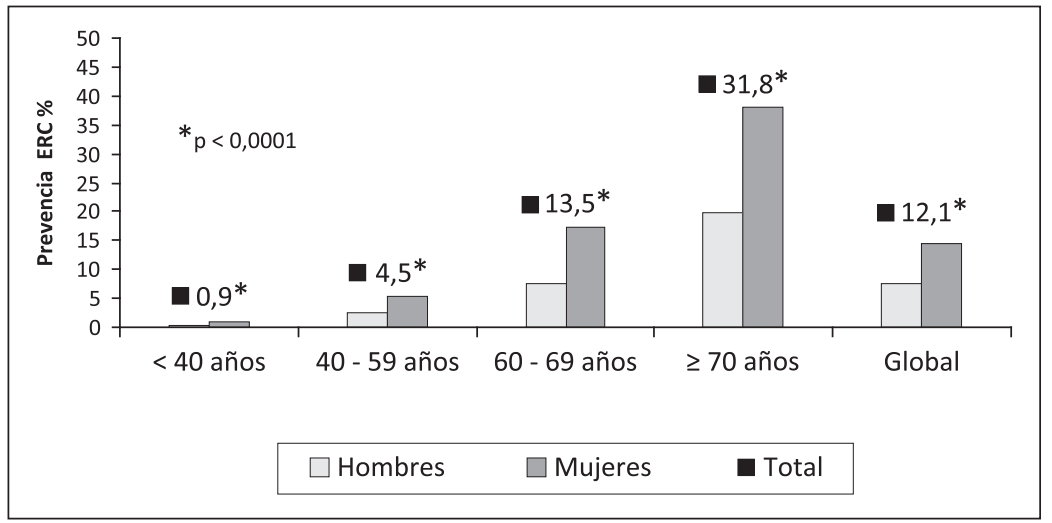

Figura 1. Prevalencia de enfermedad renal crónica (ERC) por grupos de edad y género.
$3 \mathrm{~A}$ y $3 \mathrm{~B}$ respectivamente); $2,3 \%$ en Etapa 4 y 1,3\% en Etapa 5. En cada una de las etapas las mujeres y los pacientes $\geq 60$ años tuvieron porcentajes significativamente superiores ( $\mathrm{p}<0,0001)$ (Tabla 4$)$.

Del total de pacientes con ERC, 26,8\% (905 pacientes) tenían ERC oculta, todas mujeres con un incremento progresivo y significativo en los grupos de mayor edad ( $<<0,0001)$ (Figura 2$)$.

En los 5.718 pacientes a quienes se midió simultáneamente creatinina sérica y albuminuria, hubo $777(13,6 \%)$ con ERC y $1.494(26,1 \%)$ con albuminuria $>30 \mathrm{mg} / \mathrm{g}$ (91,7\% microalbuminuria y $8,3 \%$ macroalbuminuria). En aquellos con ERC, 315 pacientes $(40,5 \%)$ tuvieron microalbuminuria, con porcentajes significativamente superiores en mujeres y en mayores de 60 años $(\mathrm{p}<0,0001)$. Hubo una débil pero significativa correlación negativa entre VFGe $<60$ y microalbuminuria (Pearson $r=-0,2, p<0,05)$. No se encontraron diferencias significativas en los porcentajes de microalbuminuria entre los grupos Etapa 3B y Etapa 4, pero si al comparar ambos con el grupo Etapa 3A (p < 0,05). La Tabla 5 muestra las características del grupo con $\mathrm{VFGe}<60$ y albuminuria según etapas de la ERC.

El 50\% de los pacientes con VFGe $<60$ se controlaban en los Programas de Salud Cardiovascular de su consultorio, pero sólo 1,1\% tenían consignado en su ficha clínica el diagnóstico de ERC.

El reporte estadístico de todos los consultorios en el período de la investigación informó una prevalencia de ERC de 1,3\%. (Cuenta anual Salud Municipal Concepción). La Figura 3 grafica las diferencias de prevalencia entre el reporte oficial y el cálculo desde los datos de VFGe en cada consultorio $(\mathrm{p}<0,0001)$.

En el análisis de regresión logística los grupos de mayor edad, el género femenino y la microalbuminuria tuvieron respectivamente $7 ; 2,4$ y 2,2 veces más riesgo de presentar VFGe $<60$ (Tabla 6). 
Tabla 4. Características de pacientes con ERC por etapas, según género y edad $<0 \geq 60$ años $(n=3.371)$

\begin{tabular}{|c|c|c|c|c|}
\hline & Etapa III A & Etapa III B & Etapa IV & Etapa V \\
\hline$N^{0}$ Pacientes/Etapa ${ }^{a}$ & $2.748(81,5)$ & $500(14,8)$ & $78(2,3)$ & $45(1,3)$ \\
\hline Hombres & $19 \%$ & $26 \%$ & $40 \%$ & $36 \%$ \\
\hline Mujeres $^{d}$ & $81 \%$ & $74 \%$ & $60 \%$ & $64 \%$ \\
\hline Edad $^{b}$ (años) & $71(12,2)$ & $76(11,7)$ & $70(13,8)$ & $64(10,9)$ \\
\hline$<60$ años & $15,9 \%$ & $8,4 \%$ & $20,5 \%$ & $28,9 \%$ \\
\hline$\geq 60$ años $^{d}$ & $84,1 \%$ & $91,6 \%$ & $79,5 \%$ & $71,1 \%$ \\
\hline VFGE $E^{b, c}$ & $54(4)$ & $39,1(3,9)$ & $23,6(4,4)$ & $8,6(2,6)$ \\
\hline
\end{tabular}

${ }^{\mathrm{a}}$ Valores expresados como número total de casos y porcentaje entre paréntesis. ${ }^{b}$ Valores expresados como media y desviación estándar entre paréntesis. 'VFGE.(MDRD-4): Filtrado glomerular estimado expresado en $\mathrm{ml} / \mathrm{min} / 1,73$. 'Las mujeres y los pacientes $\geq 60$ años tuvieron porcentajes significativamente superiores $(p<0,0001)$.

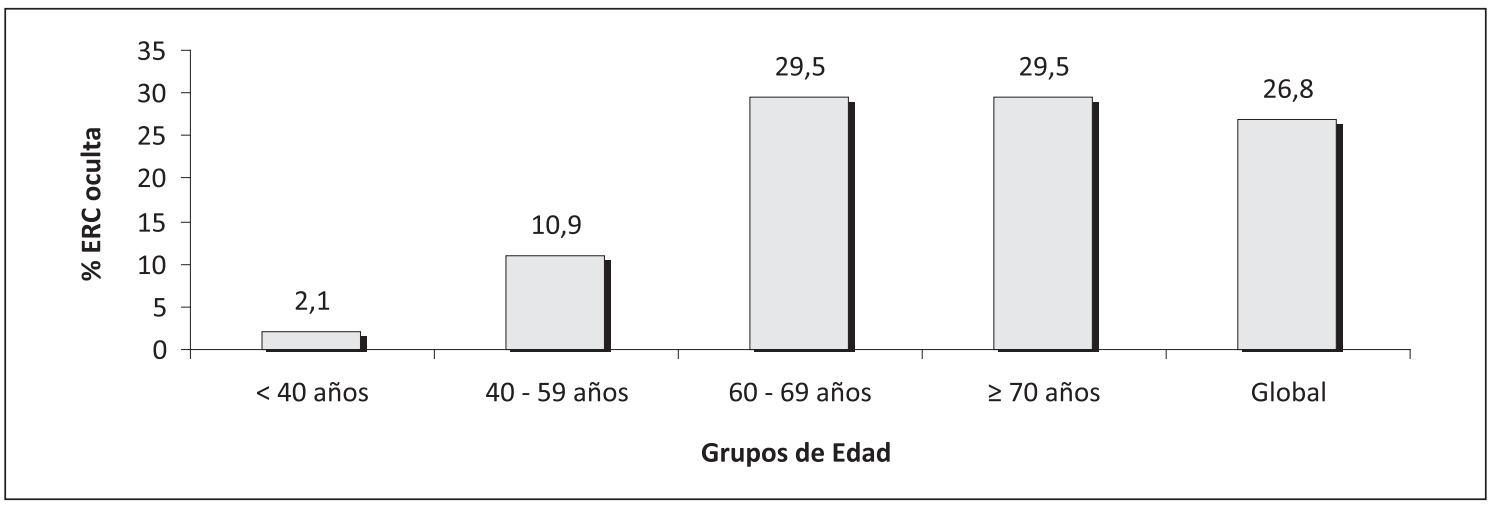

Figura 2. Prevalencia de enfermedad renal crónica (ERC) oculta por grupos de edad.

Tabla 5. Características del grupo con FGe $<60$ y Albuminuria según etapa de la ERC $(\mathbf{n}=777)$

\begin{tabular}{|c|c|c|c|}
\hline & Etapa III A & Etapa III B & Etapa IV \\
\hline Total de pacientes & 630 & 130 & 17 \\
\hline Hombres & $23 \%$ & $30 \%$ & $24 \%$ \\
\hline Mujeres & $77 \%$ & $70 \%$ & $76 \%$ \\
\hline Edad $^{a}$ & $72(10)$ & $74 \quad(10)$ & $74 \quad(10)$ \\
\hline$<60$ años & $11,3 \%$ & $8,5 \%$ & $12 \%$ \\
\hline$\geq 60$ años & $88,7 \%$ & $91,5 \%$ & $88 \%$ \\
\hline VFGE ${ }^{a, b}(M D R D-4)$ & 54 (4) & $39 \quad(4)$ & $25 \quad(4)$ \\
\hline RAC $^{a, c}$ & $63(138)$ & $113(178)$ & 192 (339) \\
\hline $\mathrm{RAC}<30 \mathrm{mg} / \mathrm{gr}$ & $64 \%^{d}$ & $40 \%$ & $41 \% \mathrm{e}^{\mathrm{e}}$ \\
\hline $\mathrm{RAC} \geq 30 \mathrm{mg} / \mathrm{gr}$ & $36 \%{ }^{d}$ & $60 \%$ e & $59 \%$ e \\
\hline
\end{tabular}

จalores expresados como media y desviación estándar entre paréntesis. ${ }^{\circ}$ VFGE MDRD-4. Filtrado glomerular estimado expresado en $\mathrm{ml} / \mathrm{min} / 1$,73. 'RAC: Razón albúmina/creatinina en orina aislada (mg/gr). ${ }^{\mathrm{d}}$ Comparación \% RAC Etapa 3A v/s Etapa 3B y 4 $\mathrm{p}<0,05$. e Comparación \% RAC Etapa 3b vs Etapa 4 NS. 


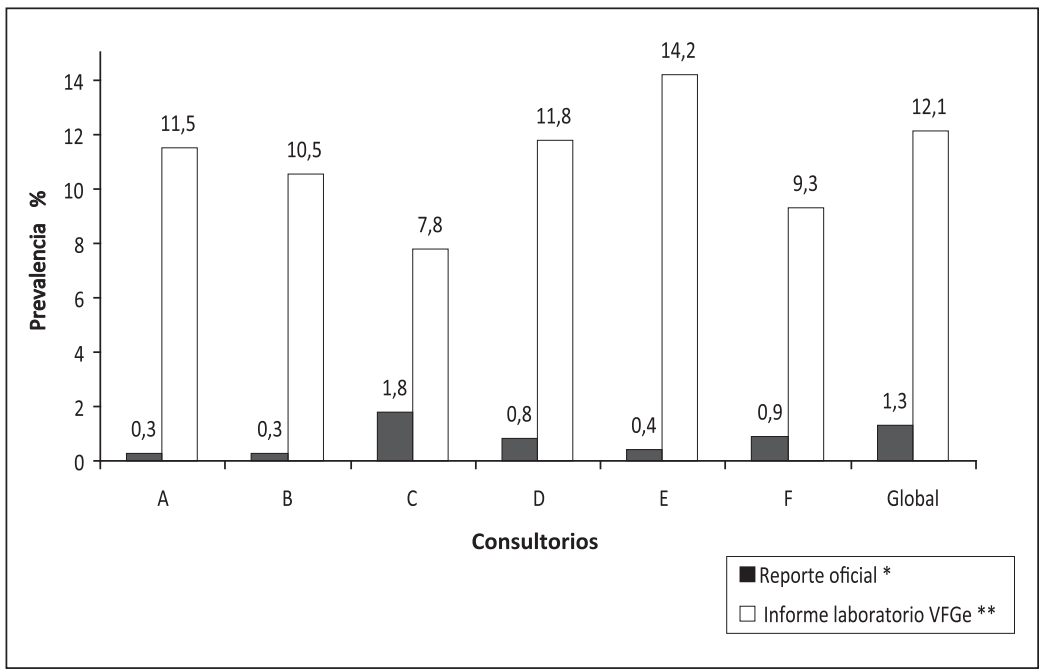

Figura 3. Prevalencia Enfermedad Renal Crónica (ERC) por consultorio. Diferencias entre el reporte oficial y el informe de VFGea ${ }^{a}$ VFGe: Velocidad de filtración glomerular estimada expresado en $\mathrm{ml} / \mathrm{min} / 1,73$. *Prevalencia de ERC. Reportes oficiales de cada consultorio. ${ }^{* *}$ Calculo de prevalencia desde los datos de VFGe en cada consultorio.

Tabla 6. Regresión logística binaria. Variable dependiente filtrado glomerular estimado $\geq 60$ vs $<60(\mathrm{ml} / \mathrm{min} / 1,73)$

\begin{tabular}{|lcccc|}
\hline Variable independiente & Beta & OR & Cl 95\% & p \\
\hline Edad $^{\text {a }}$ & 1,953 & 7,051 & $5,571-8,923$ & 0,0001 \\
\hline Género $^{b}$ & 0,906 & 2,475 & $2,066-2,965$ & 0,0001 \\
\hline Albuminuriac $^{-1,831}$ & 2,296 & $1,944-2,712$ & 0,0001 \\
\hline
\end{tabular}

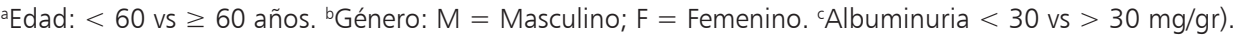

\section{Discusión}

Los resultados de este estudio aportan información epidemiológica sobre la ERC en población adulta atendida en los consultorios urbanos (CESFAM) en nuestro país. La prevalencia global de ERC fue $12,1 \%$, con mayores porcentajes en mujeres $(14,5 \%)$ y en mayores de 60 años $(23,3 \%)$, en contraste con el $2,7 \%$ en la población general reportado en la Encuesta Nacional de Salud 2009$2010^{3}$. Estas diferencias de prevalencia entre la población general y la atención primaria validarían concentrar los recursos en programas preventivos de la enfermedad en los CESFAM.

Dos estudios en España y uno en Canadá reportaron prevalencias de ERC comparables en la atención primaria de $21,3 \%, 16,4 \%$ y $15,2 \%$ respectivamente, así como también una menor VFGe en mujeres y adultos mayores ${ }^{22-24}$.

En nuestro estudio, sorprendentemente las cifras oficiales de los consultorios evaluados reporta- ron durante el mismo período una prevalencia de ERC de sólo 1,3\%. Asimismo, 50\% de los pacientes con VFGe $<60 \mathrm{ml} / \mathrm{min} / 1,73 \mathrm{~m}^{2}$ se controlaban en los Programas de Salud Cardiovascular de los consultorios, pero sólo el 1,1\% tenía consignado el diagnóstico o sospecha de ERC, aun cuando los exámenes sí estaban registrados. Esta discordancia reflejaría desconocimiento de los nuevos conceptos de la ERC en los equipos de salud de los CESFAM, lo cual junto con retrasar el diagnóstico y tratamiento oportuno genera pérdidas de tiempo y recursos económicos. Así también, dado que los pacientes con ERC clasifican en el nivel alto de riesgo cardiovascular, el no consignar el diagnóstico induciría a categorizarlos erróneamente con bajo riesgo y demorar la terapia adecuada ${ }^{21,25-26}$.

El 26,8\% de los pacientes con VFGe $<60$, especialmente mujeres y grupos de mayor edad, tuvieron creatinina sérica $\leq 1,0 \mathrm{mg} / \mathrm{dL}$, lo que definimos como ERC oculta. Este hallazgo advierte del riesgo en la indicación de fármacos o interven- 
ciones diagnóstico/terapéuticas potencialmente nefrotóxicas. Estudios similares han reportado prevalencias variables de ERC oculta entre 10,4\% y $37,3 \% 0^{22-24,27-29}$. Promover que los resultados de creatinina sérica sean informados con la VFGe de acuerdo a la ecuación MDRD-4 u otra, facilitaría la pesquisa de la ERC y contribuiría a evitar o disminuir riesgos en pacientes con ERC oculta ${ }^{16,23}$.

La edad > 60 años, el sexo femenino y la albuminuria $>30 \mathrm{mg} / \mathrm{g}$ resultaron, en el análisis de regresión logística, predictores de riesgo independientes de ERC. Ello valida la recomendación de la Guías de prevención para pesquisar la ERC en estos grupos, especialmente en presencia de otras condiciones de riesgo como HTA y DM ${ }^{16,20,25-26,30-32}$.

La mayor prevalencia de ERC observada en adultos mayores coincide con otras publicaciones, que reportan un explosivo aumento del diagnóstico en estos grupos basado sólo en la VFGe $<60$ $\mathrm{ml} / \mathrm{min}^{23,33-35}$. Ello junto con provocar inquietud y temor en los afectados, genera sobrecarga a los ser-

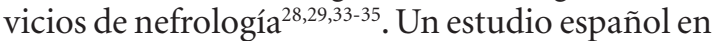
ancianos con $\mathrm{VFGe}<60$, observó que los pacientes sin proteinuria, diabetes o $\mathrm{VFGe}<30$ presentaron estabilidad de la VFGe en el tiempo, sugiriendo los autores mantener cautela en rotularlos con el diagnóstico de ERC hasta no tener mayores elementos clínicos o de laboratorio ${ }^{33}$. Algunas sociedades científicas han propuesto subdividir la Etapa 3 en 3A (VFG: $45-59 \mathrm{ml} / \mathrm{min} / 1,73 \mathrm{~m}^{2}$ ) y 3B (VFG: $30-44 \mathrm{ml} / \mathrm{min} / 1,73 \mathrm{~m}^{2}$ ), considerando las diferencias de riesgo cardiovascular entre estos subgrupos (36-37). En nuestro estudio, 81,5\% de los pacientes con VFGe $<60 \mathrm{ml} / \mathrm{min} / 1,73 \mathrm{~m}^{2}$ estuvieron en el subgrupo $3 \mathrm{~A}$ y $14,8 \%$ en el $3 \mathrm{~B}$. La coexistencia de ERC y microalbuminuria fue $40,5 \%$ y hubo significativa correlación negativa entre VFGe y albuminuria, mayor en los grupos 3B y 4. Nuevos estudios prospectivos de estos subgrupos, especialmente en aquellos sin otras manifestaciones de ERC, y la incorporación de nuevos métodos de medición y estimación de la VFG permitirán establecer el significado clínico y pronóstico de estos hallazgos ${ }^{34-38}$.

Una de las limitaciones del estudio es que no permitió discriminar una disminución transitoria del VFG de una ERC establecida, considerando que por definición se exige repetir los exámenes a lo menos en tres meses ${ }^{16}$. Asimismo, incluyó sólo a personas a quienes se solicitó creatinina sérica y albuminuria. Ambas limitaciones pudieran mi- tigarse en parte considerando el elevado número de pacientes estudiados ( $35 \%$ total de la población adulta asignada a los consultorio), y que 58,5\% de ellos estaban registrados en el programa cardiovascular de su CESFAM, donde se controlan las dos patologías con mayor riesgo de desarrollar la enfermedad, diabetes mellitus e hipertensión arterial. Con estas salvedades, nuestro trabajo aporta una primera aproximación sobre la prevalencia de la ERC en la atención primaria.

Otra limitación fue la utilización de la ecuación MDRD-4, considerando las diferencias existentes en los procedimientos calibrados de medición de la creatinina sérica entre laboratorios, la falta de estandarización con el laboratorio de referencia donde se desarrolló la ecuación y la inexistencia de estudios que la validen en nuestra población ${ }^{17}$. El sesgo de la calibración de la creatinina afecta la precisión en el cálculo de la VFGe especialmente en valores $>60 \mathrm{~mL} / \mathrm{min} / 1,73 \mathrm{~m}^{2}{ }^{17}$. La influencia de estas consideraciones en nuestros resultados se atenuaron debido a que las mediciones se realizaron en un solo laboratorio, lo cual disminuyó el sesgo asociado a la variabilidad de la técnica y porque el estudió sólo incluyó a pacientes con VFG $\leq$ a $60 \mathrm{~mL} / \mathrm{min} / 1,73 \mathrm{~m}^{2}$, que es el valor donde la ecuación MDRD-4, presenta mayor aproximación y exactitud con los valores de filtrado glomerular medido con ${ }^{125}$ I-Iotalamato $^{17}$.

Finalmente, nuestro estudio reveló un alto número de personas con ERC no diagnosticada, especialmente en el Programa Cardiovascular, lo cual evidenciaría desinformación de las Guías Clínicas de Prevención y Manejo de la ERC $16,21,26,31,32,36,37$. La entrega de Guías o la capacitación ocasional de los equipos de salud, no garantiza su adecuada implementación, pues ellas requieren un período de internalización para incorporar nuevos conceptos, métodos diagnósticos y recomendaciones terapéuticas $^{39-40}$. Algunos factores que pudieran explicar lo señalado serían la excesiva carga asistencial de los equipos, la escasa o nula comunicación entre el nivel primario y los nefrólogos de referencia, las múltiples guías de otras especialidades y la alta rotación de médicos en los consultorios.

Un trabajo cooperativo entre los CESFAM y la nefrología hospitalaria con visitas tutoriales de un nefrólogo a los consultorios, podría mejorar la pesquisa y tratamiento de la ERC, potenciar la educación continua a los equipos de salud y disminuir las listas de espera en el nivel secundario ${ }^{39,40}$. 
En conclusión: Los resultados de este estudio mostraron que la ERC tiene una prevalencia importante en la población atendida en la atención primaria, principalmente en mujeres y adultos mayores. Sin embargo, permanece en general subdiagnosticada especialmente en los programas de salud cardiovascular.

Agradecimientos: Los autores agradecen al personal del Laboratorio Clínico y la Unidad de Estadísticas de la Dirección de Salud, Municipalidad de Concepción, por su invaluable trabajo en proporcionar información esencial para este estudio.

\section{Referencias}

1. Annual Data Report 2010 de Unites States Renal Data System. Disponible en: www.usrds.org/adr.htm. [Consultado el 2 de febrero 2011].

2. Registro de la Sociedad Española de Nefrología. Disponible en: www.senefro.org/ [Consultado el 2 de febrero 2011].

3. Encuesta Nacional de Salud 2009-2010. Ministerio de Salud Chile. Disponible en:_www.redsalud.gov.cl/portal/url/item/99c12b89738d80d5e04001011e0113f8.pdf [Consultado el 2 de febrero 2011].

4. Poblete H. XXIV Cuenta de Hemodiálisis Crónica en Chile 2010. Sociedad Chilena de Nefrología. Registro Diálisis. Disponible en: http://www.asodi.cl/joomla/ archivos/cuenta\%20XXX\%20HDC\%202010.pdf [Consultado el 2 de febrero 2011].

5. ERA-EDTA Registry. Disponible en: www.era-edta-reg. org/index.jsp? $\mathrm{p}=13$. [Consultado el 2 de febrero 2011].

6. Indicadores básicos de Salud 2007.Ministerio de Salud de Chile. Disponible en: http://deis.minsal.cl/deis/indicadores/indi2007.pdf. [Consultado el 2 de febrero 2011].

7. Bakris GL, Ritz E. Hypertension and Kidney Disease. A Marriage that Should Be Prevented. Kidney Int 2009; 75: 449-52.

8. Di Angelantonio E, Chowdhury R, Sarwar N, Aspelund T, Danesh J, Gudnason V. Chronic kidney disease and risk of major cardiovascular disease and non-vascular mortality: prospective population based cohort study. BMJ 2010; 341: 4986-93

9. Smith GL, Lichtman JH, Bracken MB, Shlipak MG, Phillips CO, Di Capua P, et al. Renal impairment and outcomes in heart failure: systematic review and metaanalysis. J Am Coll Cardiol 2006; 47: 1987-96.

10. Coresh J, Selvin E, Stevens LA, Manzi J, Kusek JW, Eggers $\mathrm{P}$, et al. Prevalence of chronic kidney disease in the
United States. JAMA 2007; 298: 2038-47.

11. Go AS, Chertow GM, Fan D, McCulloch CE, Hsu CY. Chronic kidney disease and the risks of death, cardiovascular events, and hospitalization. N Engl J Med 2004; 351: 1296-305.

12. Brugts JJ, Knetsch AM, Mattace-Raso FU, Hofman A, Witteman JCM. Renal function and risk of myocardial infarction in an elderly population: the Rotterdam study. Arch Intern Med 2005; 165: 2659-65.

13. Van Biesen W, De Bacquer D, Verbeke F, Delanghe J, Lameire N, Vanholder R. The glomerular filtration rate in an apparently healthy population and its relation with cardiovascular mortality during 10 years. Eur Heart J 2007; 28: 478-83.

14. World Kidney Day 2011. Disponible en: http://www. worldkidneyday.org/page/press-material. [Consultado el 2 de febrero 2011].

15. Levey AS, Bosch JP, Lewis JB, Greene T, Rogers N, Roth D. A more accurate method to estimate glomerular filtration rate from serum creatinine: a new prediction equation. Modification of Diet in Renal Disease Study Group. Ann Intern Med 1999; 130: 461-70.

16. Levey AS, Coresh J, Balk E, Kausz AT, Levin A, Steffes MW, et al. National Kidney Foundation Practice Guidelines for Chronic Kidney Disease: Evaluation, Classification, and Stratification. Ann Intern Med 2003; 139: 137-47.

17. Gracia S, Montañes R, Bover J, Cases A, Deulofeu R, Martin de Francisco AL, et al. Documento de Consenso: Recomendaciones sobre la utilización de ecuaciones para la estimación del filtrado glomerular en adultos. Nefrología 2006; 26 (6): 658-65.

18. Ginsberg JM, Chang BS, Matarese RA, Garella S. Use of single voided urine samples to estimate quantitative proteinuria. N Engl J Med 1983; 309: 1543-6.

19. Zelmanovitz T, Gross JL, Oliveira JR, Paggi A, Tatsch M, Azevedo MJ. The receiver operating characteristics curve in the evaluation of a random urine specimen as a screening test for diabetic nephropathy. Diabetes Care 1997; 20: 516-9.

20. Methven S, MacGregor MS, Traynor JP, Hair M, O’Reilly DS, Deighan CJ. Comparison of urinary albumin and urinary total protein as predictors of patient outcomes in CKD. Am J Kidney Dis 2011; 57 (1): 21-8.

21. National Kidney Foundation. K/DOQI clinical practice guidelines for chronic kidney disease: evaluation, classification, and stratification. Am J Kidney Dis 2002;39: Suppl 1: S1-S266.

22. De Francisco AL, De la Cruz JJ, Cases A, De la Figuera M, Egocheaga MI, Górriz JI, et al. Prevalencia e insuficiencia renal en centros de Atención Primaria de España. 
Estudio EROCAP. Nefrología 2007; 27: 300-12.

23. Lou Arnal LM, Campos Gutiérrez B, Boned Juliani B, Turón Calzada JM, Gimeno Orna JA. Estimation of glomerular filtration rate in primary care: prevalence of chronic kidney disease and impact on referral to nephrology. Nefrologia 2008; 28 (3): 329-32.

24. Duncan L, Heathcote J, Djurdjev O, Levin A: Screening for renal disease using serum creatinine: who are we missing ?. Nephrol Dial Transplant 2001; 16 (5): 1042-6.

25. Sarnak MJ, Levey AS, Schoolwerth AC, Coresh J, Culleton B, Hamm LL, et al. American Heart Association Councils on Kidney in Cardiovascular Disease, High Blood Pressure Research, Clinical Cardiology, and Epidemiology and Prevention. Kidney disease as a risk factor for development of cardiovascular disease: a statement from the American Heart Association Councils on Kidney in Cardiovascular Disease, High Blood Pressure Research, Clinical Cardiology, and Epidemiology and Prevention. Circulation. 2003; 108: 2154-69.

26. Implementación del enfoque de riesgo en el Programa de Salud Cardiovascular. Ministerio de Salud Chile. Disponible en: www.redsalud.gov.cl/portal/url/item/78 7e4765248bc9e0e04001011f0172b5.pdf. [Consultado el 2 de febrero 2011].

27. Rodrigo MP, Andrés MR. Detección de insuficiencia renal oculta en consulta primaria mediante la aplicación de la ecuación MDRD-abreviada: análisis de 1.000 pacientes. Nefrología 2006; 26 (3): 339-43.

28. Labrador PJ, Mengotti T, Jiménez M, Macías M, Vicente F, Labrador J, et al. Occult renal failure in primary care. A women's problem?. Nefrología 2007; 27 (6): 716-20.

29. Redón J, Gil V, Cea-Calvo L, Lozano JV, Martí-Canales JC, Llisterri JL, et al. The impact of occult renal failure on the cardiovascular risk stratification in an elderly population: the PREV-ICTUS study. Blood Press 2008; 17 (4): 212-9.

30. De Jong PE, Gansevoort RT. Fact or fiction of the epidemic of chronic kidney disease-let us not squabble about estimated GFR only, but also focus on albuminuria. Nephrol Dial Transplant 2008; 23: 1092-5.

31. Flores JC, Alvo M, Borja H, Morales J, Vega J, Zúñiga C, et al. Enfermedad renal crónica: Clasificación, identificación, manejo y Complicaciones. Rev Med Chile 2009; 137: 137-77.

32. Ministerio de Salud 2008. Guía Clínica Prevención Enfermedad Renal Crónica. AUGE. Disponible en: http://webhosting.redsalud.gov.cl/minsal/archivos/ guiasges/2008/GPC_Prevencion_ERC2008.pdf. [Consultado el 2 de febrero 2011].

33. Heras M, Fernández-Reyes MJ, Guerrero MT, Sánchez R, Muñoz A, Macías M.C, et al. Ancianos con enfermedad renal crónica: ¿qué ocurre a los 24 meses de seguimiento?. Nefrología 2009; 29 (4): 343-9.

34. Glassock RJ, Winearls CG. Routine reporting of estimated glomerular filtration rate: not ready for prime time. Nature Clinical Practice Nephrology 2008; 4 (8): 422-3.

35. Glassock RJ, Winearls CG. An epidemic of chronic kidney disease: fact or fiction? Nephrol Dial Transplant 2008; 23: 1117-21.

36. Guías NICE. BMJ 2008; 337:1530-45.

37. The Renal Association Guidelines. Disponible en: www.renal.org/Clinical/GuidelinesSection/Guidelines. aspx. [Consultado el 2 de febrero 2011].

38. Coresh J, Stevens LA, Levey AS. Chronic kidney disease is common: What do we do next?. Nephrol Dial Transplant 2008; 23: 1122-5.

39. McClellan WM, Knight DF, Karp H, Brown WW. Early detection and treatment of renal disease in hospitalized diabetic and hypertensive patients: important differences between practice and published guidelines. Am J Kidney Dis 1997; 29: 368-75.

40. Pommer W, Bressel F, Chen F, Molzahn M. There is room for improvement of preterminal care in diabetic patients with end-stage renal failure--the epidemiological evidence in Germany. Nephrol Dial Transplant 1997; 12: 1318-20. 